Werkes wurde zugleich eine mit mehreren, bis zu $500 \mathrm{~m}$ langen Tunnels und weiteren Kunstbauten versehene Fahrstraße von Ayent bis Tseuzier erstellt, welche natürlich auch der Bauernsame und dem Tourismus zugute kommt. Ayent ist von Sitten aus ohnehin seit langem mit Wagen erreichbar. Aber die Besucherzahl wird künftig noch stark wachsen, wenn einmal die geplante Rawilstraße erstellt sein wird. Diese im schweizerischen Nationalstraßennetz vorgesehene Querverbindung durch die Berneralpen, soll vom Obersimmental, d. h. von Lenk her durch das Iffigental aufsteigen, dann in einem 4,4 km langen Scheiteltunnel in $1790 \mathrm{~m}$ den Rawil passieren und am Lac de Tseuzier wieder zutage treten. Hier wird ein Ast der neuen Straße über deri Staudamm des Sees und in die bestehende Straße nach Ayent geführt, also die Verbindung mit Sitten geschaffen. Ein zweiter Ast aber soll an der Staumauer abzweigen, auf der linken Flanke des Liène-Tales weiterleiten nach Montana-Vermala, der beliebten Sonnenterrasse, $1000 \mathrm{~m}$ über dem Rhonetal. Von hier aus besteht die Straßenverbindung nach Sierre bereits. So wird also der Lac de Tseuzier künftig am Durchgangsverkehr liegen und dann wohl einiges von seiner stillen Romantik verlieren.

\title{
HONGKONG, DIE BRITISCHE HAFENSTADT IM FERNEN OSTEN
}

HANS BERNHARD

Wer von Hinterindien über das Südchinesische Meer nach Hongkong fliegt, bemerkt in der Nähe der chinesischen Küste fast plötzlich das Auftauchen vieler kleiner und kleinster Inseln. Ihre scharf gebuchteten Küsten und das steile Eintauchen ins Meer vermitteln den Eindruck einer typischen Senkungslandschaft. Es ist denn auch ein Teil des Südchinesischen Berglandes, das um große Beträge abgesunken ist, und obwohl Terrassen auch von deutlicher Hebung zeugen, dominieren doch die ertrunkenen Täler, die von der Wasserscheide der westlich gelegenen Insel Lantau nach Osten und Südosten untertauchen.

Mitten in diesem Insellabyrinth ist Hongkong dank seiner günstigen Lage und seiner geschichtlichen Bevorzugung zu ganz besonderer Bedeutung gelangt. Trotz der Nähe zu China und seiner alten Kultur ist die Geschichte von Hongkong jung und kurz. Vor der britischen Okkupation war es eine verlassene Insel mit wenig Fischern und Piraten. Im Opiumkrieg 1839-42 wurde der noch wüste und leere, aber verkehrsgünstige, geschützte und genügend tiefe $\mathrm{Hafen}$ als Basis für die britische Marine benützt. Damit beginnt seine eigentliche Geschichte.

Hongkong, die $1013 \mathrm{~km}^{2}$ große britische Kronkolonie umfaßt die gleichnamige bergige Insel mit der Stadt Viktoria ( $80 \mathrm{~km}^{2}$ groß, seit $18+2$ britisch) und die nördlich davon liegende Halbinsel Kowloon ( $10 \mathrm{~km}^{2}$, seit 1860 britisch). Flächenmäßig am größten, aber nur dünn besiedelt sind die New Territories mit $923 \mathrm{~km}^{2}$, die Großbritannien im Jahre 1898 pachtweise erwarb. Dazu gehören das Hinterland von Kowloon bis zur Höhe der Deep Bay sowie 75 Inseln.

Hongkongs Bedeutung beruht in allererster Linie auf seinem Hafen. Er gehört zu den größten der Welt. In gewissen Jahren steht er sogar nach der Zahl der ein- und ausfahrenden Schiffe an erster Stelle. Der $43,5 \mathrm{~km}^{2}$ große Hafen liegt zwischen der Nordseite der Insel Hongkong und dem gegenüberliegenden Festland. Er hat zwei $\mathrm{Zu}$ gänge, von denen der östliche nur 450 Meter breit ist, aber eine Fahrrinne von über 18 Metern Tiefe aufweist. Der westliche Eingang ist fast 5 Kilometer weit, aber bedeutend seichter. Er wird durch verschiedene Inseln vor den wichtigsten Winden geschützt. Die zwischen Hongkong und Kowloon eingeengten Gezeitenströme haben im inneren Hafen eine Fahrrinne geschaffen, die auch größten Schiffen die Durchfahrt ermöglicht. Als im September 1945 die britische Flotte den Hafen nach der japanischen Invasion wieder besetzte, lagen hier über 100 Schlachtschiffe aller Größen, bis zum 40000 Tonnen schweren Duke of York.

Da die Gezeitenunterschiede nur 2 bis $2 \frac{1}{2} 2$ Meter betragen, sind keine teuren Dockanlagen nötig. Auf der West- und Ostseite der Halbinsel liegen 12 Tiefwasserbecken, 


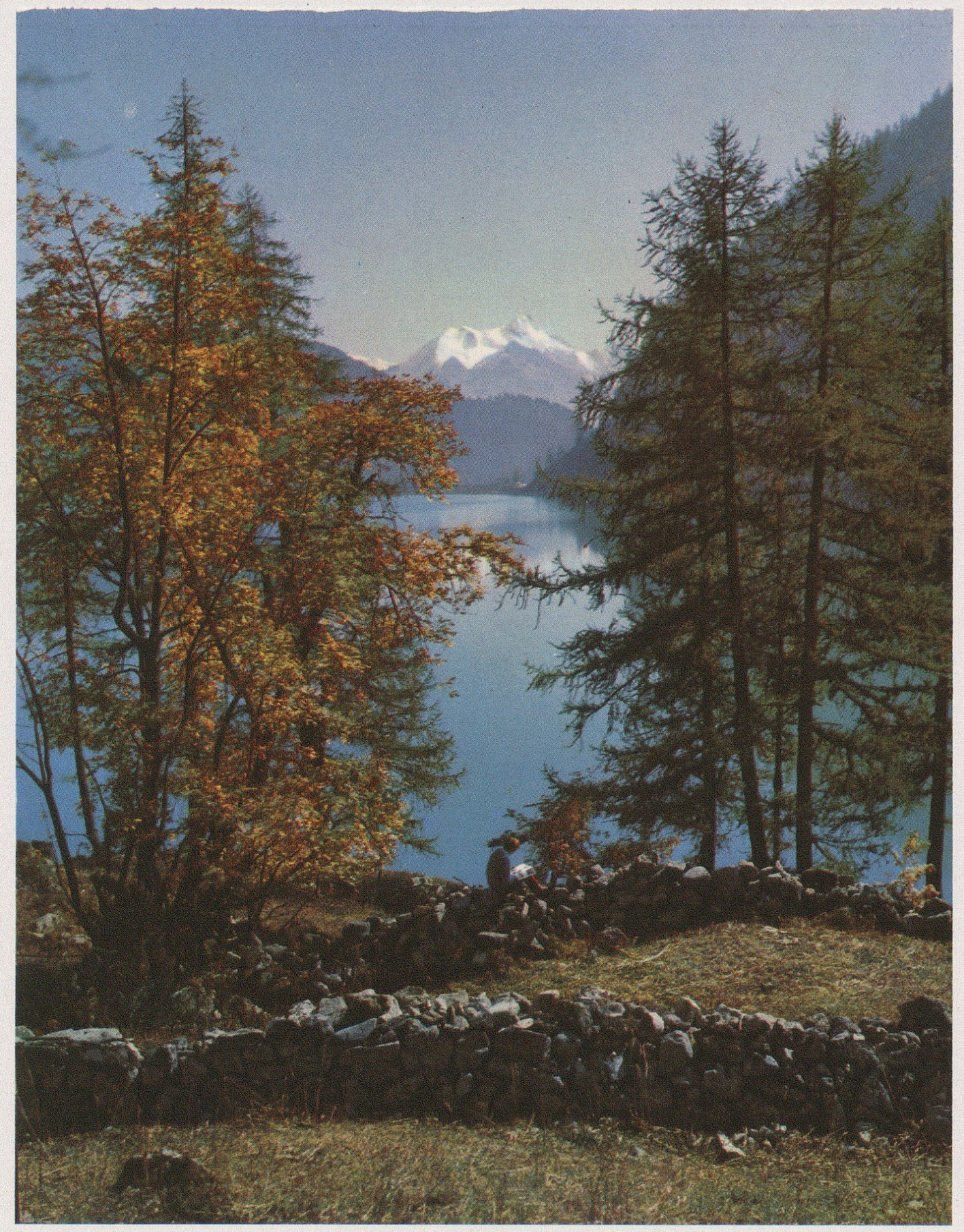

Lac de Tseuzier. Im Hintergrund Weißhorn (Walliser Alpen).

Photo Zufferey, UVT, Sion 


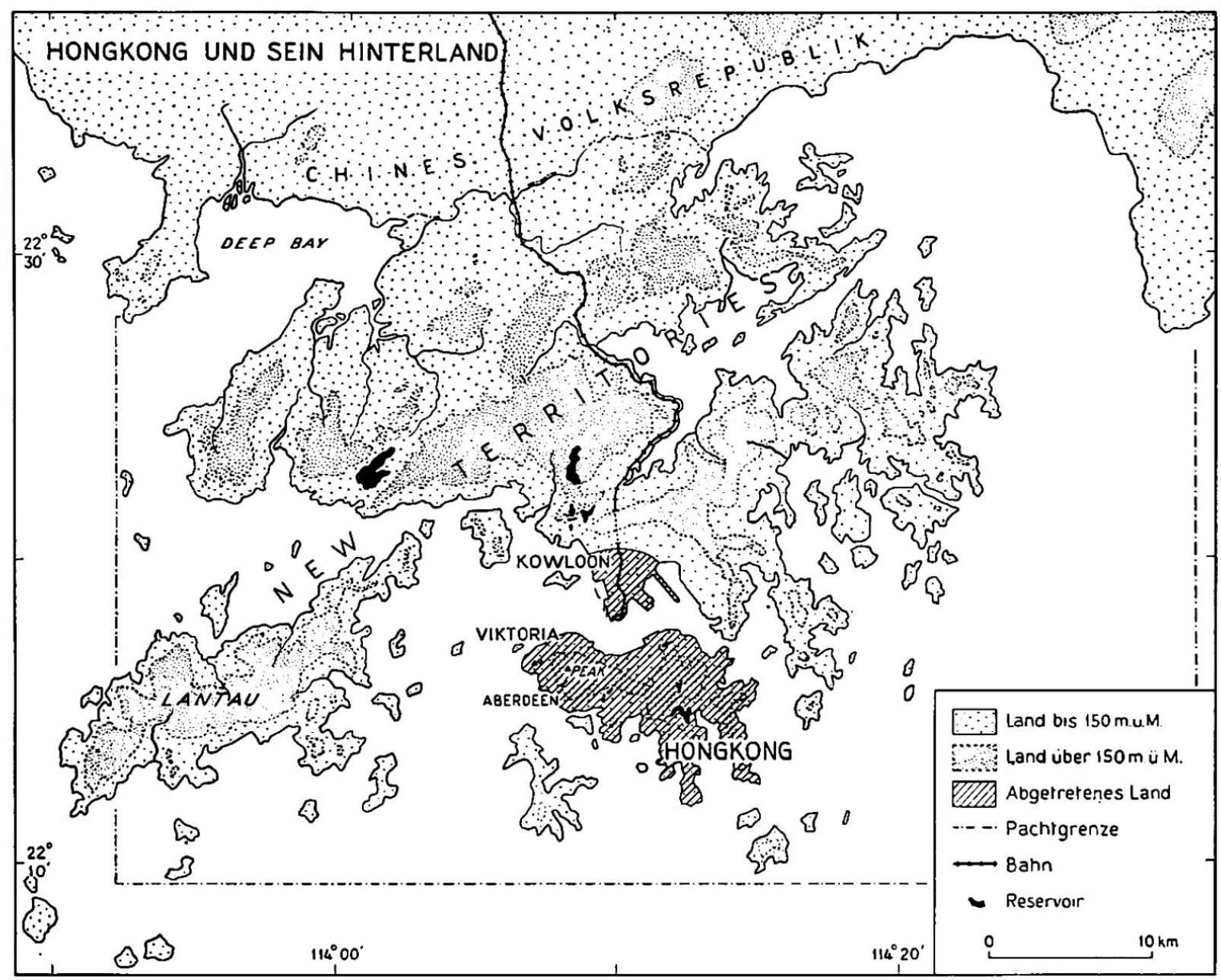

Fig. 1. Lage von Hongkong

vor Hongkong deren 2; aber auch im inneren Hafen gibt es über 40 Ankerplätze für das Ausladen von Großschiffen von denen oft über 100 im Hafen liegen und um die es von Tschunken und Barken geradezu wimmelt. - Im Schutze der benachbarten Inseln sind die Wasser außerordentlich ruhig. Einzig beim Durchgang eines Taifuns droht den Schiffen auch hier Gefahr. Von durchschnittlich 25 Taifunen, die den Hafen jährlich passieren, fallen die meisten auf die Monate Juli bis Oktober. Sie entstehen im Gebiet der Karolinen und setzen sich in parabelartiger Kurve in nordwestlicher Richtung fort. Sie erreichen Geschwindigkeiten von über $500 \mathrm{~km}$ im Tag. Ihr schmales Zentrum, dessen starker Druckgradient die heftigen Winde erzeugt und das nur etwa $80 \mathrm{~km}$ Durchmesser hat, streicht zwar selten direkt über Hongkong hinweg. Durch einen gut organisierten Warnungsdienst werden Heftigkeit und Zeit der Ankunft dieser Wirbelstürme rechtzeitig mitgeteilt. Für Tschunken und kleinere Schiffe ist je ein mit starken Molen versehener Taifunhafen auf der Nordostseite von Hongkong und im Westen Kowloons errichtet worden (siehe Fig. 3). Größere Schiffe fahren in die Nordwestecke des Hafens, der fast immer genügend Schutz bietet. Katastrophal waren die Folgen des Taifuns vom September 1937: 11000 Personen ertranken, 1255 Tschunken und Kleinschiffe waren gesunken und von 101 Ozeanschiffen 28 gestrandet. - Einer Zeitungsnotiz von 11. Juni 1960 ist zu entnehmen, daß der Taifun «Mary» (böse Stürme erhalten immer Frauennamen), der mit rund 170 Stundenkilometern über die Kolonie hinwegfegte, 64 Todesopfer forderte und 18000 Personen obdachlos machte. 150 Schiffe, zumeist Fischerboote, sollen vermißt werden.

Neben den günstigen örtlichen Verhältnissen verdankt der Hafen von Hongkong seine ungeahnte Entwicklung der glänzenden Verkehrslage vor den Toren Südchinas. 


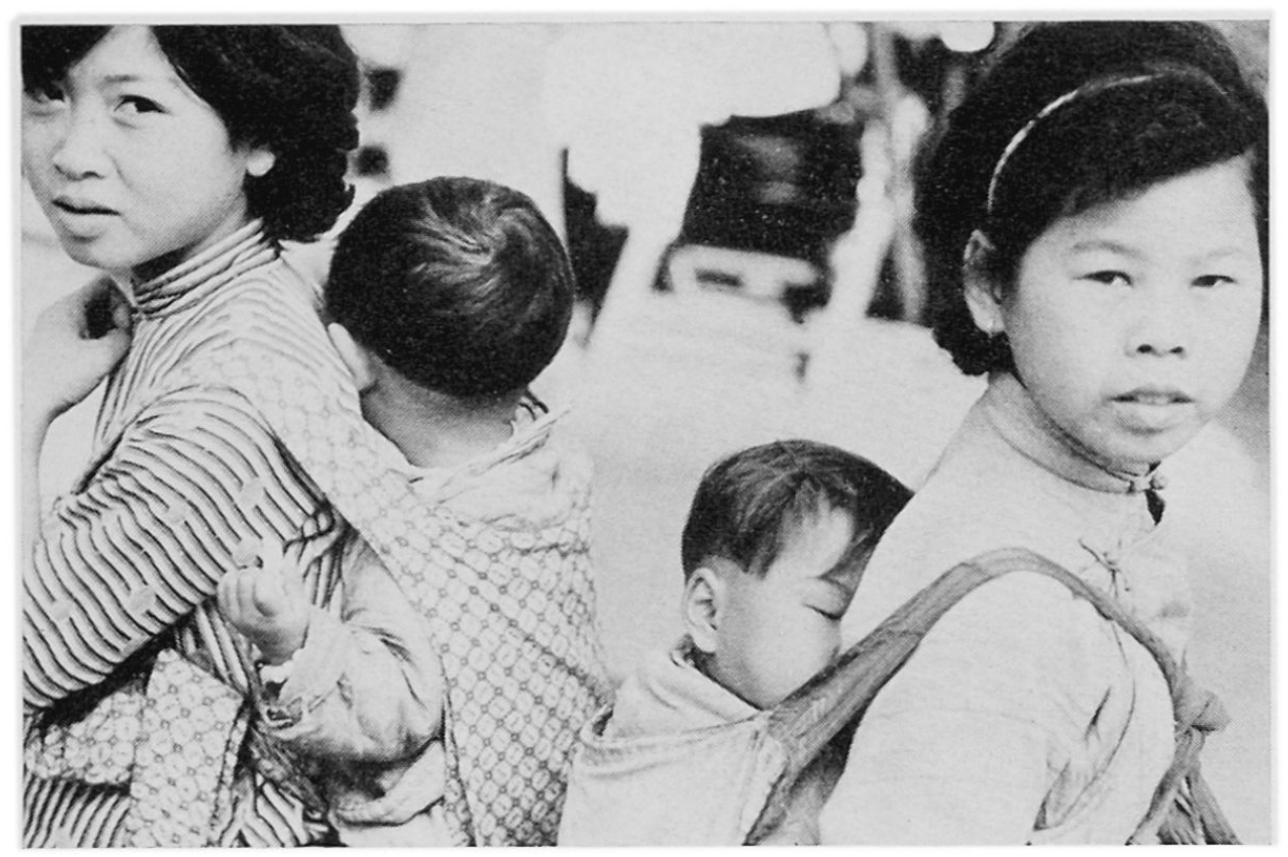

Fig. 2. Schon sieben- bis achtjährige Mädchen tragen ihre kleinen Geschwister den ganzen Tag auf dem Rücken herum. Eine einfache Vorrichtung verhindert das Herausfallen der Kleinkinder und gibt den Mädchen möglichst große Bewegungsfreiheit.

Er liegt am östlichen Eingang des Si-Kiang, der weit ins Landesinnere führt. Auf einer Strecke von $1600 \mathrm{~km}$ gibt es keinen Zugang von ähnlicher Bedeutung. Vor dem Zwciten Weltkrieg gingen denn auch $43 \%$ des Exportes von Hongkong ins nördliche Hinterland. Seit der Bildung der Rotchinesischen Republik ist dieser Verkehr stark zurückgegangen. Kleine Küstenschiffe (selten über 300 Tonnen) fahren flußaufwärts nach Kanton oder längs der chinesischen Küste. Der Hauptverkehr geht aber nach Ländern außerhalb Chinas; nach Thailand, Indonesien, Vietnam, Südkorea, Japan. Ganz allgemein hat die Kolonie von der chinesischen Revolution stark profitiert. Viele westliche Handels- und Schiffahrtsgesellschaften haben ihren Sitz von chinesischen Häfen nach Hongkong verlegt, und viel Flüchtlingskapital kam nach der Stadt, deren Güterumschlag vor allem auf Kosten von Schanghai und Tientsin gewaltig angewachsen ist. Er beträgt bereits mehr als sechs Mio Tonnen. Aber nur 20\% der eingeführten Waren bleiben in der Kolonie. Hongkong ist Freihafen, ohne Taxen und Zölle, mit minimalen Hafengebühren. Die Lagerverhältnisse sind sehr günstig, Kapital für irgendwelche Finanzaktion ist überall erhältlich. Treibstoff für Schiffe sowie Proviant für Mannschaften und Passagiere sind leicht zu beschaffen. Verschiedene Industrien dienen der Veredlung von Rohstoffen und Halbfabrikaten. Schon seit den ersten Jahren nach der Gründung der Kolonie wurden Handel und Verkehr im Hafen durch einen vom Gouverneur ernannten Hafenmeister kontrolliert und geleitet, während der Bau der Piers, Docks und Lagerhäuser (letztere mit weit über 1 Mio t Fassungsvermögen) ganz der privaten Initiative überlassen sind. Drei große, europäische und fünf kleinere, chinesische Werften machen den Schiffsbau zur bedeutendsten Industrie von Hongkong. Erstere bauen Schiffe bis zu 10000 Tonnen und können selbst große Ozeandampfer reparieren.

Mit dem Hafen ist auch die Stadt in stürmischem Tempo angewachsen. Das heweisen schon die Bevölkerungszahlen: Vor dem Zweiten Weltkrieg zählte Hongkong 1 1 12 Mio Einwohner. Während der japanischen Besetzung verblieben nur noch 750000 Leute in der Kolonie. Seit der Rückkehr der Briten hat die Bevölkerung um 300\% zugenommen, und Hongkong nähert sich der Dreimillionengrenze. - Von wo kommen diese enormen Volksmassen und wie finden sie auf dem verhältnismäßig kleinen 


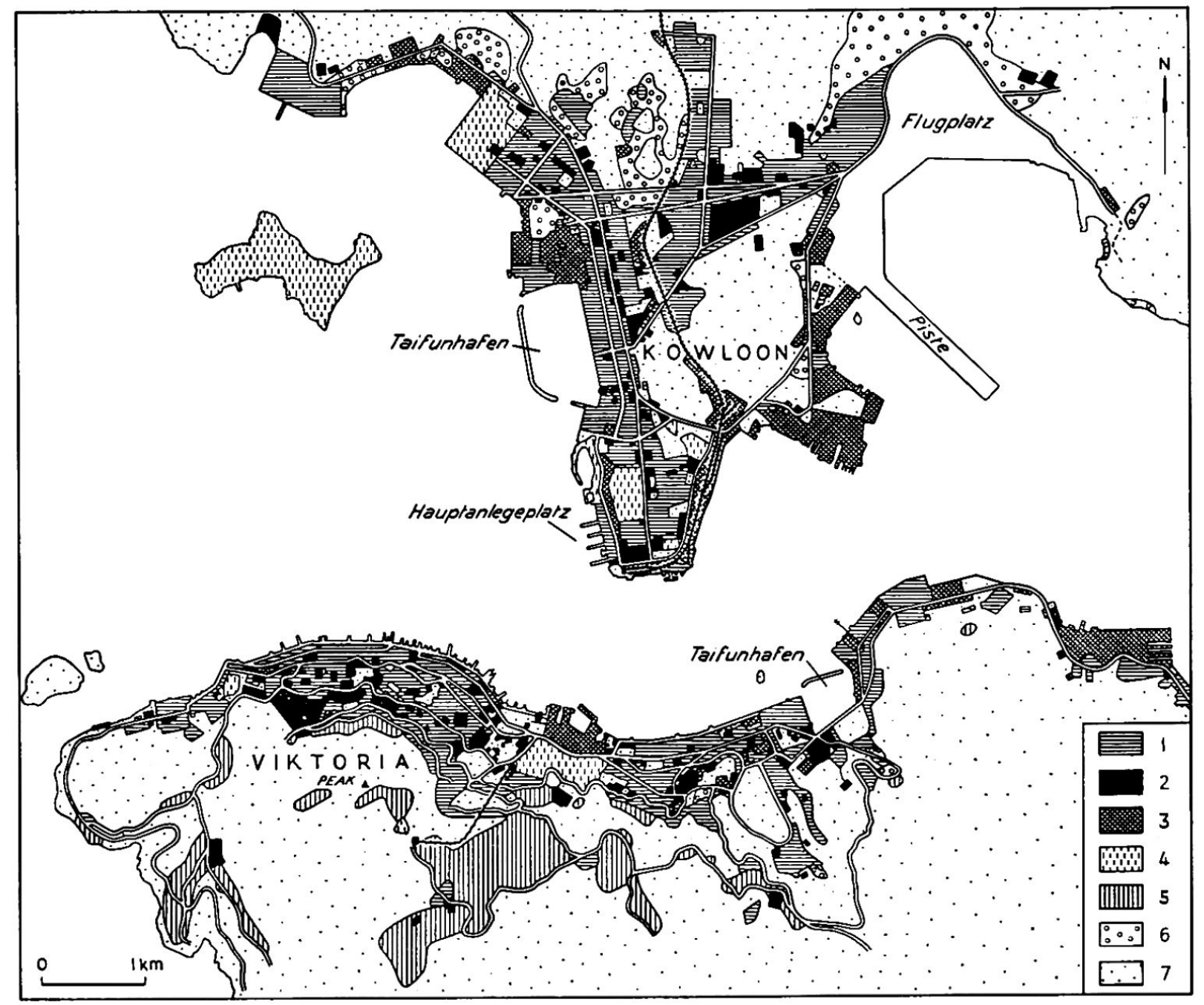

Fig. 3. Wirtschaftsgefüge von Hongkong. 1 Wohnzonen und Läden, 2 Handels- und Verwaltungsquartiere, 3 Industrie- und Verkehrsanlagen, 4 Militäranlagen, 5 Streu-Wohnquartiere, 6 Landwirtschaftsgebiete, 7 Unüberbaute Gebiete mit Flüchtlingsquartieren.

Raum ihren Platz? 99\% der Einwohner Hongkongs sind Chinesen. Die Fruchtbarkeit dieser Rasse zeigt sich dem Besucher auf Schritt und Tritt. Tausende von Frauen fast aller Altersstufen tragen ihre Kinder und schon kleine, 7 bis 8 jährige Mädchen ihre Geschwister den ganzen Tag auf dem Rücken herum. Auch die Rudel spielender Kinder auf allen Straßen und Plätzen zeugen vom enormen Nachwuchs der Chinesen. Damit allein läßt sich aber die große Zunahme der Bevölkerung doch nicht erklären. Fast ebenso wichtig ist die Zuwanderung. Die Zahl der meist illegalen Flüchtlinge aus Rotchina soll eine Million überschreiten. Im Gesamten nimmt die Bevölkerung der Stadt jährlich um 250000 Menschen zu. - Für die ganze Kolonie ergäbe sich heute eine mittlere Volksdichte von 3000 Einwohnern pro $\mathrm{km}^{2}$. Doch sind die Verhältnisse noch viel extremer.

Von der $10 \mathrm{~km}^{2}$ großen Halbinsel Kowloon sind nur $7 \mathrm{~km}^{2}$ besiedelt; denn große Gebiete im Osten und Norden sind der ungünstigen Boden- oder Reliefverhältnisse wegen noch nicht überbaut. Die Volksdichte für dieses Gebiet beträgt 80000 Einwohner $/ \mathrm{km}^{2}$. Alt Kowloon-City, einst eine typische, ummauerte Chinesenstadt ist in den letzten 20 Jahren drastisch umgestaltet worden und hat sich zur eigentlichen Geschäftsstadt mit durchaus europäischem Stil entwickelt. Verkaufsläden aller Art für Einheimische und Fremde mit schreienden Lichtreklamen in chinesischer Schrift, Cáfés, Unterhaltungslokale, Gaststätten und Hotels flankieren die zum Teil breiten Straßen in Schachbrettlage. Verkehrspolizisten und Signalanlagen meistern den Verkehr der 


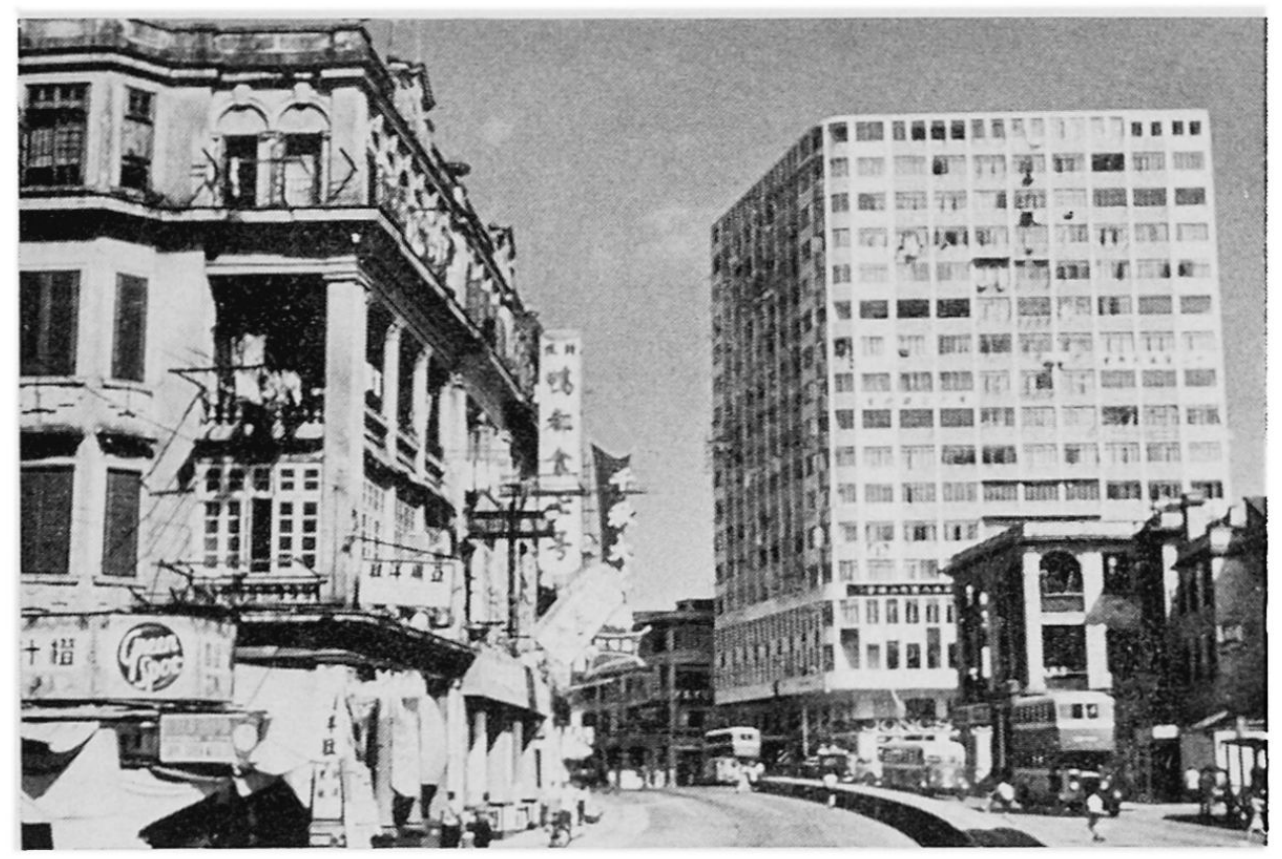

Fig. 4. Jordan Road in Kowloon. Nördlich des Taifunhafens quert sie in west-östlicher Richtung die Halbinsel. Modernste Hochbauten stehen neben älteren Wohn- und Geschäftshäusern.

zweistöckigen Autobusse und Trams, die die wichtigen Straßen in ununterbrochener Folge und raschem Tempo befahren. Daneben hat aber auch der Kuli seine Bedeutung für Menschen- und Gütertransport absolut nicht verloren, und er ist bemüht, seine Kunden nicht nur billig, sondern auch rasch zu bedienen. - Von der Südspitze der Halbinsel führt eine Bahn nach der rotchinesischen Grenze und von dort weiter ins Landes. innere. Während der Neujahrstage 1960 (chinesisches Neujahr ist der 28. Januar) konnten während zwei Tagen und zwei Nächten vor dem Bahnhof lange Menschenkolonnen beobachtet werden, welche schwerbepackt auf den nächsten Zug warteten, um ihre Verwandten in Rotchina zu besuchen. - Im Nordosten der Halbinsel liegt einer der größten Flugplätze der Welt und vor drei Jahren ist eine moderne Landepiste weit in die Kowloon Bay hinausgebaut worden (siehe Fig. 3).

Viktoria, die Hauptstadt der Insel Hongkong, die durch einen großartigen Fährenbetrieb mit Kowloon verbunden ist, hat sogar eine Volksdichte von 140000 Einwohnern $/ \mathrm{km}^{2}$. Im zentralen Teil der Stadt liegen die Kontore der europäischen Geschäfte, Banken und Verkehrsgesellschaften und hangwärts gegen den $600 \mathrm{~m}$ hohen Peak mit 4 bis 6 Grad kühleren Sommertemperaturen, die Administrationen der Regierung. Den westlichen Teil Viktorias bildet das Chinesenviertel. Es ist ein Labyrinth von schmal gewundenen Gassen, eine bunte Mischung kleiner Geschäfte und offener Verkaufsstände, Einraumwerkstätten und überfüllter Wohnhäuser. Es sind ärmliche, oft baufällige, eng zusammengebaute Häuser mit unhaltbaren sanitären Verhältnissen. In einer Wohnung für 10 Personen wohnen oft mehr als 60 Chinesen, ja sogar Verandas und Flachdächer werden vermietet.

Im Osten der Stadt haben rund 300000 Flüchtlinge ihre Wohnungen in den Berg geschlagen und mit Holz, Dachpappe und Blechkanistern ausgekleidet und sich so zun Teil recht wohnlich eingerichtet; denn die Chinesen sind im allgemeinen sauber und ordnungsliebend. Aber das Wasser muß oft weit zugetragen werden, und in einem winzigen Raum wohnen 8 bis 10 Personen. Weitere 300000 Chinesen leben auf Wohnbooten im nordwestlichen Hafenbecken und in Aberdeen, einem Fischerdorf auf der Südseite der Insel. In dichter Fülle reiht sich Boot an Boot, von der massiven Barke bis zum baufälligen. Schiffchen, das nur ungenügend gegen Regen und Kälte schützt. Fahrende Händler versorgen die Leute mit den notwendigsten Lebensmitteln. Die 


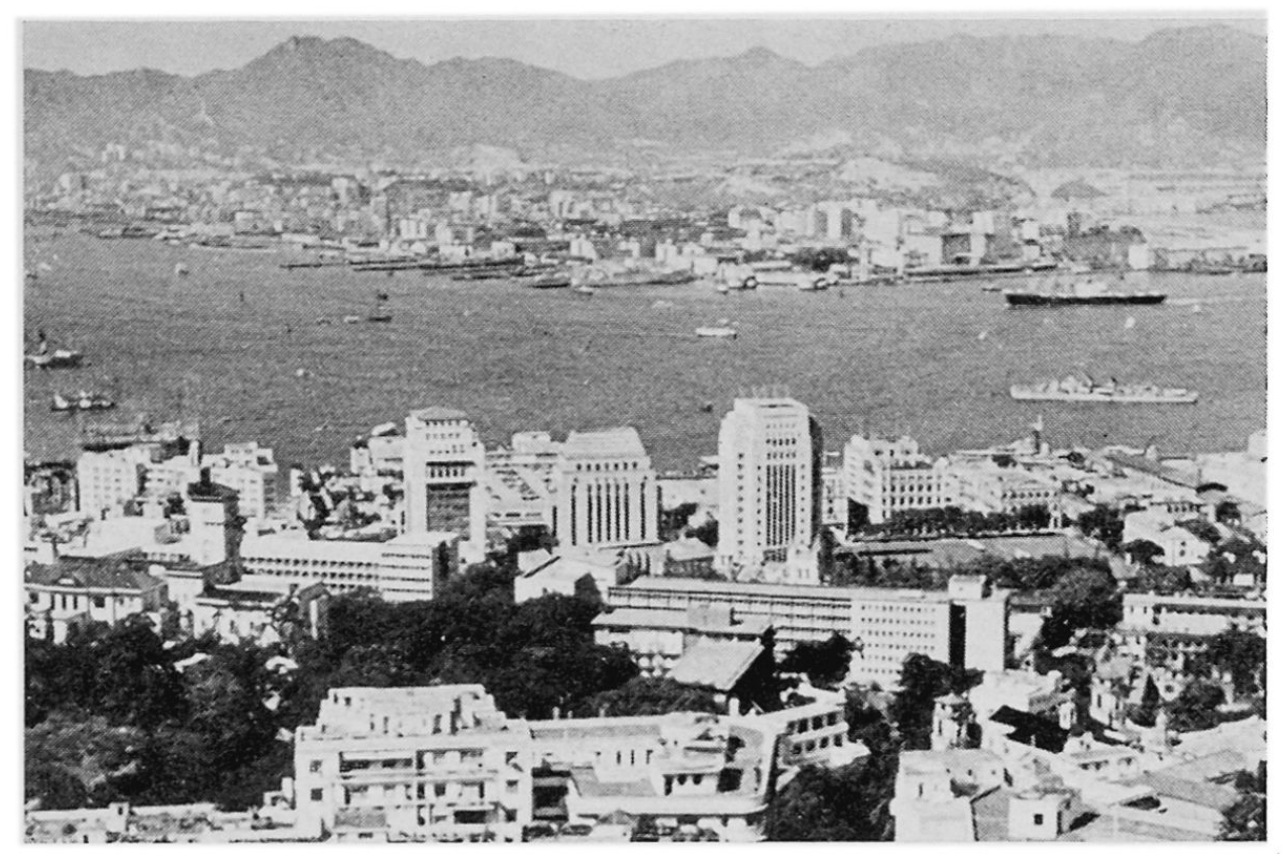

Fig. 5. Blick vom Peak auf das Administrations- und Geschäftsviertel von Viktoria. Die Bildmitte zeigt den westlichen Teil des Hafens und dahinter die Halbinsel Kowloon. Der Gebirgszug im Hintergrund gehört schon zu Rotchina.

Männer verdienen sich etwas Geld mit fischen, handwerklicher Arbeit oder als Kulis verschiedenster Art.

Die Regierung versucht diese katastrophale Wohnungsnot zu beheben. Seit 1954 ist sie vom Bau kleiner Zweifamilienhäuschen zur Erstellung großer Wohnblöcke übergegangen. Diese siebenstöckigen Bauten können etwa 4000 Personen aufnehmen. Die beiden Längsschenkel des $\mathrm{H}$-förmigen Baues sind auf jedem Stockwerk in $12 \mathrm{Woh}$ nungen unterteilt. Diese bestehen aus einem Raum von 3 auf 71/2 Metern, der vom Mieter weiter ausgestaltet werden kann. Die Quertrakte enthalten die gemeinsamen Waschräume, Bäder und Toiletten. Im Erdgeschoß befinden sich die verschiedenen Werkstätten und Arbeitsräume und auf dem Flachdach spielen die Kinder. In solchen Wohnblöcken sind bis jetzt 400000 Personen untergebracht worden. Dennoch ist es fraglich, ob die Regierung den Wettlauf mit dem gewaltigen Bevölkerungszuwachs gewinnen wird, müssen doch gleichzeitig auch Schulhäuser, Spitäler, Kinderheime und viel Ähnliches eingerichtet werden.

Auch die Wasserversorgung der Stadt ist außerordentlich prekär. Schon 1863, als die Bevölkerung von Hongkong die 100000 Grenze überschritten hatte, wurde in der Nähe des Peak das erste Reservoir mit einem Fassungsvermögen von 90000 hl erstellt. Acht Jahre später mußte es bereits auf 3 Mio hl vergrößert werden. Seither sind immer wieder neue und größere Stauseen gebaut worden, auf der Insel, in Kowloon und besonders im Gebiet der New Territories (siehe Fig. 1). Erwähnt seien nur Wongneichong auf Viktoria (23 Mio hl), Jubilee im Norden der Halbinsel (135 Mio hl) und Tai Lam Chung im Südwesten der New Territories (300 Mio hl). Da aber die Niederschläge hauptsächlich mit dem Südwestmonsun zwischen Mai und September fallen, sind die 15 Reservoirs während der Wintermonate fast trocken; der Wasserbezug ist während dieser Zeit streng auf 4 Stunden pro Tag rationiert. Bereits sind Verhandlungen mit Rotchina im Gang, um vom gebirgigen Hinterland jenseits der Grenze unverbrauchte Reserven zu nutzen.

Hongkong gedeiht. Stadt, Hafen und Bevölkerung entfalten sich explosionsartig. Wer versorgt diese riesige Menschenmasse mit den notwendigen Nahrungsmitteln, wird man fast beängstigt fragen. Da sind einmal die New Territories, dieses $923 \mathrm{~km}^{2}$ 


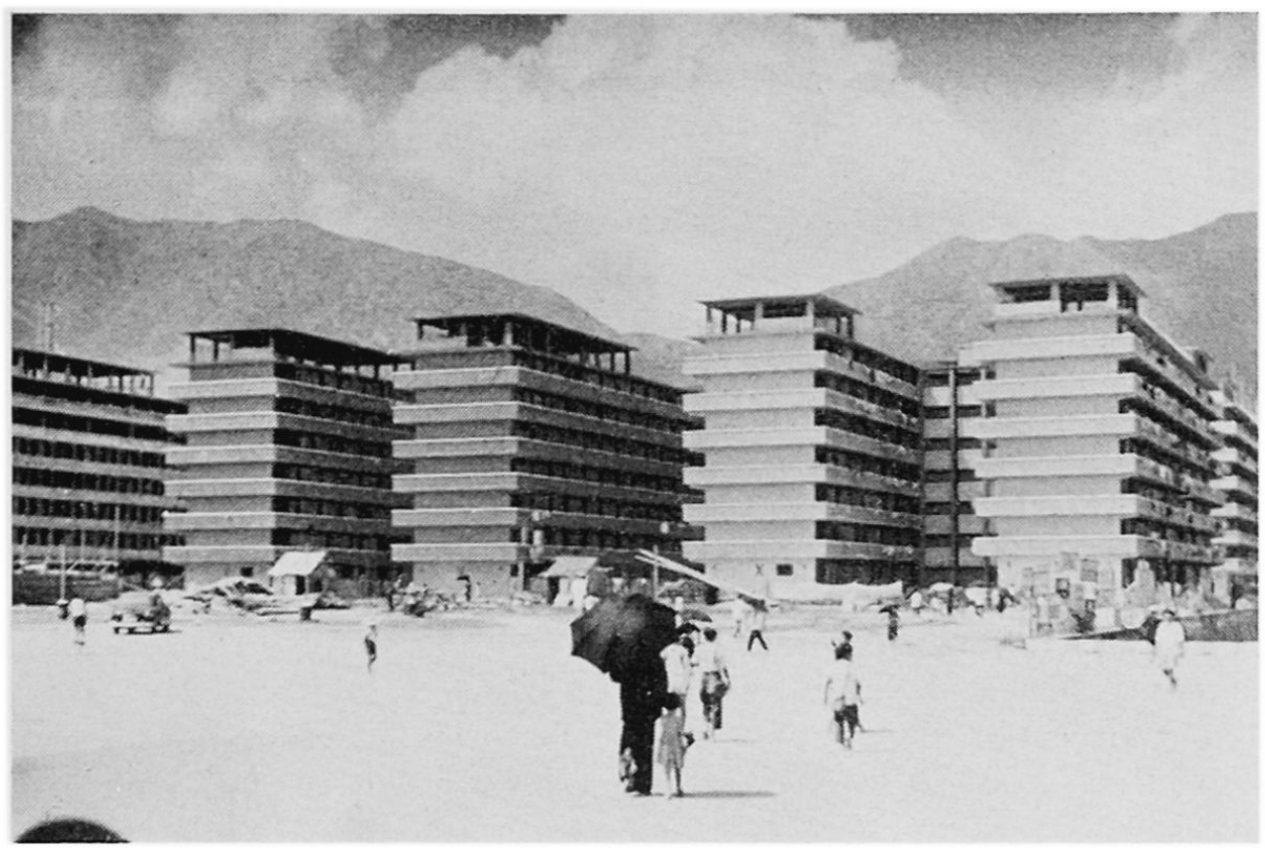

Fig. 6. Wohnblockkolonien, die bis zu 40000 Flüchtlinge aufnehmen können, sind vor allem im Gebiet der Halbinsel Kowloon errichtet worden.

große Pachtgebiet, das 1997 zurückgegeben werden muß. Sie bilden das agrarische Hinterland der Kolonie. Zwischen höheren Granitrücken liegen ausgeräumte Sedimentzonen, wenige Meter über Meer, in Küstennähe, mit recht fruchtbaren Böden, im Ganzen etwa $200 \mathrm{~km}^{2}$. Das subtropische Klima mit zum Teil stark maritimem Einschlag ermöglicht den Anbau von Agrumen, Bananen, Ananas, Papayas, Feigen, Aprikosen, Pfirsichen und Tee. An erster Stelle, mit 72\% der Anbaufläche steht der Reis, der, in Saatbeeten aufgezogen, in 100 Tagen reift und oft ein zweites oder gar drittes Mal angepflanzt werden kann. Im Herbst beim Eintritt kühlerer Witterung wird auch noch viel Gemüse geerntet. Die Viehzucht ist nur unbedeutend. Dieses dünnbesiedelte Agrargebiet, das an einzelnen Orten auf eine mehr als 1000-jährige, ununterbrochene Besiedlung zurückblicken kann, mit noch alten Anbaumethoden und bäuerlichen Verhältnissen wie vor Generationen, bildet ein wichtiges Ver乏orgungsgebiet für die aufstrebende Stadt. Doch genügt die eigene Produktion immer weniger und ansehnliche Mengen an Lebensmitteln müssen eingeführt werden. Einzig mit Fischen kann sich Hongkong selbst versorgen.

Indem die Kolonie immer mehr über ihre Lebensmittelbasis hinauswuchs, mußte sie zwangsläufig zur Industriezone werden. Auch hier vollzog sich die Wandlung in unglaublich kurzer Zeit. Die Chinesen sind ein handwerklich begabtes Volk und waren schon recht früh in Kleinbetrieben aller Art tätig. Aber in knapp 20 Jahren sind 5000 Fabriken aus dem Boden geschossen und fast jede Woche wird wieder ein neuer Großbetrieb eröffnet. Die Kolonie besitzt zwar keine Rohstoffe, doch können diese billig zugeführt werden. Die unerhörte Zähigkeit der zugewanderten Chinesen (Überseer und Flüchtlinge) haben den Aufbau neuer Fabriken begünstigt und die konkurrenzlos billigen Arbeiter den Export nach vielen Ländern ermöglicht. Ein gelernter Arbeiter ver. dient 4 bis 12 Schweizerfranken, ein Hilfsarbeiter die Hälfte und ein ungelernter Arbeiter 2 bis 5 Franken im Tag. Der Chinese arbeitet werktags und sonntags, häufig in drei Schichten zu acht Stunden, oft aber viel länger. Nur am Neujahr (28. Januar) setzt er für ein paar Tage aus. Diese ungeheure Arbeitskraft, begünstigt von einer weitsichtigen Kolonialverwaltung, die dem freien Unternehmertum möglichst viel Spielraum läßt, hat Hongkong zum gefürchteten Exporteur gemacht, der sogar frühere Lieferanten in ihrem eigenen Lande bedroht. Auch im $\mathrm{Handel}$ wird viel verdient. Die 
Gewinnmarchen sind zwar klein, aber der Umsatz entsprechend groß. So werden jährlich für 80 bis 100 Mio Franken billige Schweizeruhren eingeführt und von hier nach Südkorea, Borneo und den Rin-Kiu-Inseln weiterverkauft.

Die Stadt finanziert sich selbst. Die Steuern sind zwar sehr klein. Ein mittlerer Angestellter bezahlt etwa $1 \%$ seines Lohnes, die meisten überhaupt nichts. Trotzdem verwandelt sich seit Jahren das jeweils budgetierte Defizit in einen Überschuß und vor wenigen Monaten wurde der Grundstein zu einem hypermodernen Stadthaus gelegt, das 20 Mio Schweizerfranken kosten wird.

Hongkong gedeiht. Immer neue Wohnblöcke schießen aus dem Boden. In 5 bis 6 Jahren sind sie dank der dichten Besiedlung amortisiert. Moderne Hotels, große Geschäftshäuser, Fabriken und Spitäler sind im Bau, Hügel werden abgetragen und seichte Meersbuchten aufgeschüttet. Der moderne Verkehr nimmt rapid zu. Der Hafen als Umschlagsplatz zwischen Europa und Asien sowie im Verkehr mit Japan, Thailand, Indonesien, Vietnam und Südkorea bewältigt von Jahr zu Jahr größere Gütermengen. Die Bevölkerungszahl schnellt beängstigend in die Höhe.

Hongkong blüht, als ob sein politisches Schicksal überhaupt nicht zur Diskussion stünde. Und doch - nur 20 bis $30 \mathrm{~km}$ weiter im Norden lauert China, liegt die rotchinesische Grenze, hermetisch abgeschlossen und streng bewacht. Die vielen Cars, die die Touristen durch das Gebiet der New Territories fahren, nähern sich ihr nur auf Distanz. In 36 Jahren muß dieses Gebiet laut Vertrag zurückgegeben werden. Damit ginge der größte Teil der Lebensmittel- und Süßwasserversorgung für die Kolonie verloren. Wird Rotchina überhaupt so lange zuwarten? Die Macht liegt ganz auf seiner Seite, und das halbe Dutzend englischer und amerikanischer Kriegsschiffe, die in Hafen von Hongkong verankert liegen, werden China keinen zu großen Eindruck machen!

\section{QUELLEN}

Davis S. G.: Hong Kong in its geographical setting. - WINKLER E. u. a.: China, «Die Erde», Band II, S. 542. - Hongkong, in «focus》 vom 3. November 1953. - GAUTsCHI H.: Reportagen im Radio Beromünster, 1960.

\section{HONGKONG}

Avant l'occupation anglaise durant la Guerre de l'opium (1839-42) Hongkong était une ile déserte avec quelques pêcheurs et pirates. Aujourd'hui cette colonie anglaise comprend $1013 \mathrm{~km}^{2}$, dont $923 \mathrm{~km}^{2}$ reviendront à la Chine Rouge en 1997.

L'importance de Hongkong se fonde premièrement sur son port, qui possède toutes les qualités d'un port moderne et compte parmi les meilleurs du monde. De temps en temps des typhons causent de gros dégâts. Comme le port, la ville a pris une grande extension. Aujourd' hui elle compte environ trois millions d'habitants. Le surplus de la population et la constante immigration de la Chine Rouge entraîne un manque d'habitations. Le gouvernement de la ville se trouve dans l'impossibilité d'y remédier. De grands buildings contenant quelques milliers d'habitants transforment de plus en plus le visage de la ville. Plus d'un million d'habitants vivent sur des bateaux ou dans des grottes qu'ils creusent à la périphérie. L'approvisionnement en eau est aussi précaire que le problème du logement. Malgré quinze grands réservoirs, l'eau est rationnée quatre heures par jour en hiver, durant la période de sécheresse.

\section{ZUR METHODIK DES GEOGRAPHIEUNTERRICHTS AN HANDELSSCHULEN}

\section{KURT BÖSIGER}

Nach dem neuen schweizerischen Reformplan für Handelsmittelschulen gehört die Geographie als Konzentrationsfach der Wirtschafts-, Rechts- und politischen Wissenschaften zur zentralen Fächergruppe und ist stundenmäßig gut dotiert. Es dürfte so- 